\title{
Search for lepton flavor violating muon decay: latest result from MEG
}

\author{
Y. Uchiyama* \\ ICEPP, The University of Tokyo, 7-3-1 Hongo, Bunkyo 113-0033, Japan \\ E-mail: uchivamadicepp.s.u-tokyo.ac.ip
}

\begin{abstract}
We present a preliminary result of a search for the lepton flavor violating decay $\mu^{+} \rightarrow \mathrm{e}^{+} \gamma$ based on data taken in the second year run of the MEG experiment at Paul Scherrer Institut in Switzerland. In 2009, MEG conducted two months data acquisition using $6 \times 10^{13}$ muon decays and stateof-the-art detectors in stable condition. We have performed a likelihood analysis with a sensitivity of $6.1 \times 10^{-12}$, which is twice better than the present upper limit set by the previous experiment, and set an upper limit on the branching fraction $\mathscr{B}\left(\mu^{+} \rightarrow \mathrm{e}^{+} \gamma\right) / \mathscr{B}\left(\mu^{+} \rightarrow \mathrm{e}^{+} \nu \bar{v}\right)<1.5 \times 10^{-11}$ at $90 \% \mathrm{CL}$.
\end{abstract}

The Xth Nicola Cabibbo International Conference on Heavy Quarks and Leptons, October 11-15, 2010

Frascati (Rome) Italy

\footnotetext{
${ }^{*}$ Speaker.

${ }^{\dagger}$ for the MEG collaboration.
} 


\section{Introduction}

Lepton flavors are conserved in the standard model of particle physics (SM). This is a consequence of absence of neutrino masses, and there is no explicit gauge symmetry for it. Definitive observations of neutrino oscillations imply finite neutrino masses and hence new physics where the lepton flavors are no longer conserved. However, any charged lepton flavor violation (cLFV) has not been observed so far. The cLFV processes are highly suppressed in the minimum extension of the SM with finite but tiny neutrino mass and are out of experimental reach.

If there are additional degrees of freedom with which the lepton flavors mix in this new sectors, cLFV could be induced. Many theories introduce such additional degrees of freedom at highenergy scale, and among them several models such as supersymmetric grand unified theories predict large enhancement in rates of cLFV processes possibly as large as the current experimental limits [四][]]. An observation of cLFV process would be a clear indication of some new physics, while improving limits would give stringent constrains on such models.

The decay $\mu^{+} \rightarrow \mathrm{e}^{+} \gamma$ is one of the most historical and stringent channel of cLFV search. Its experimental limit is $\mathscr{B}<1.2 \times 10^{-11}(90 \% \mathrm{CL})$ set by the MEGA experiment [[]]. The MEG experiment [四] started physics data taking at Paul Scherrer Institut (PSI) in 2008 aiming at the further search of the decay. Its first result based on the initial three months data was presented in [可]. In this paper, we present a preliminary result based on MEG data collected in 2009.

\section{Experimental principle}

The event signature of $\mu^{+} \rightarrow \mathrm{e}^{+} \gamma$ decay in the rest frame of decaying muon is characterized by a simple two-body decay kinematics. The positron and photon are coincident in time, emitted back-to-back, and each of them has an energy of half of muon mass, $52.8 \mathrm{MeV}$. To use this kinematic signature, we experimentally use positive muons stopped in material. Using positive muons prevents nucleus in the material from forming muonic atoms.

There are two kinds of background. One is a physics background from radiative muon decays, $\mu^{+} \rightarrow \mathrm{e}^{+} v_{\mathrm{e}} \bar{v}_{\mu} \gamma$ (RD), It becomes a prompt background when the two neutrinos carry off little energy. However, the branching fraction to such phase space is highly suppressed. The other is accidental overlaps of a high-energy positron from normal muon decay, $\mu^{+} \rightarrow \mathrm{e}^{+} v_{\mathrm{e}} \bar{v}_{\mu}$ (Michel decay) with an uncorrelated high-energy gamma ray from $\mathrm{RD}$, annihilation-in-flight of positron or bremsstrahlung. The rate of the accidental one can be written as $R_{a c c} \propto\left(R_{\mu}\right)^{2} \cdot f_{e} \cdot f_{\gamma} \cdot\left(\Delta \Theta_{e \gamma}\right)^{2}$. $\Delta t_{e \gamma}$ [目], where $R_{\mu}$ is instantaneous rate of muon beam; $f_{e}$ and $f_{\gamma}$ are positron and gamma ray background yield at signal region which are shown in Figure $\mathrm{W}$ and $\mathrm{Z}$, respectively; $\Delta \Theta_{e \gamma}$ and $\Delta t_{e \gamma}$ are resolutions of angle and timing measurements, respectively. At our muon rate of $\sim 3 \times 10^{7} / \mathrm{sec}$ and with our detector resolutions, the accidental one becomes dominant. The key of the experiment to suppress the accidental background are a good quality of continuous positive muon beam and precision detectors for positron and gamma-ray with spatial, temporal and energy resolutions.

\section{Experimental setup}

MEG is conducted at the $\pi \mathrm{E} 5$ beamline in PSI accelerator facilities. This beamline provides the world's most intense surface muons from the $590 \mathrm{MeV}$ proton ring-cyclotron at a current of 


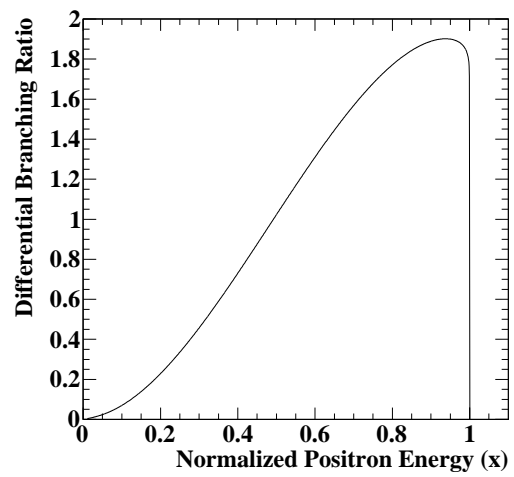

Figure 1: Positron energy spectrum of Michel decay. Positron background yield at the signal energy $(x \approx 1)$ is abundant.

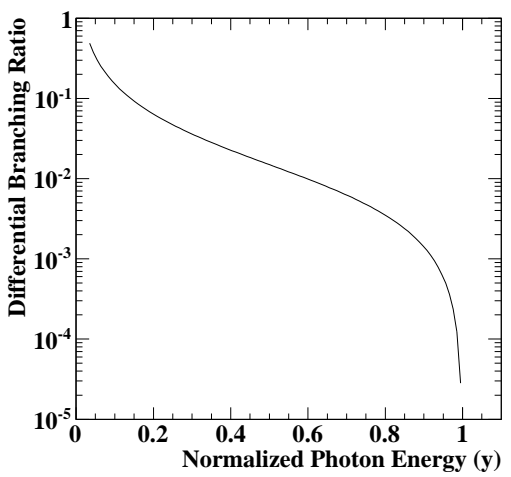

Figure 2: Gamma ray energy spectrum from RD decay. The yield at the signal region $(y \approx 1)$ is highly suppressed.

$2.2 \mathrm{~mA}$. The primary proton beam has a time structure of $50 \mathrm{MHz}$. It is fast enough compared to the muon lifetime, resulting in constant decay rate of muon which is the best in background suppression point of view. The surface muons are transported with being purified, focused and degraded through the MEG beam transport system consisting of a Wien filter, triplet magnets and beam transport solenoid, and finally stopped on a thin target composed of $205 \mu \mathrm{m}$ polyethylene/polyester sheet.

The MEG detector consists of a superconducting spectrometer and a liquid xenon (LXe) detector as shown in Figure [3. It covers about $10 \%$ of solid angle. Positrons are measured by the spectrometer, called COBRA (COnstant-Bending-RAdius), consisting of a superconducting magnets specially designed to form a highly graded magnetic field, a set of drift chamber system (DC) to measure the trajectory of positrons in the field and two sets of timing counters (TC) with plastic scintillators. The gradient field enables us to efficiently measure high-rate positrons by a preferential acceptance to high momentum as well as by quickly sweeping particles away from the tracking volume. The corresponding gamma rays emitted in the opposite side are detected by the LXe detector. The excellent properties of LXe such as high stopping power, high light yield, fast response and homogeneity fit the requirement of our gamma ray detector. It measure gamma ray energy, timing and position at the same time with 900-liter LXe as a scintillation medium and 846 photomultipliers directly immersed in LXe.

\section{Run and performance in 2009}

Before starting run in 2009, we solved a severe problem on DC operation which caused significant degradation of the spectrometer performance in 2008. During the run in 2009, all DC modules were in operation. Meanwhile, the xenon was purified during the maintenance period resulting in a $45 \%$ increase in light yield compared to that in 2008. No purification was performed during the run, and achieved more stable condition. We conducted 43 days data acquisition in November and December 2009. The beam intensity was adjusted for the muon stopping rate to be $2.8 \times 10^{7} / \mathrm{sec}$. In total, $6 \times 10^{13}$ muons were provided, and $22 \mathrm{M}$ triggered events were acquired. 

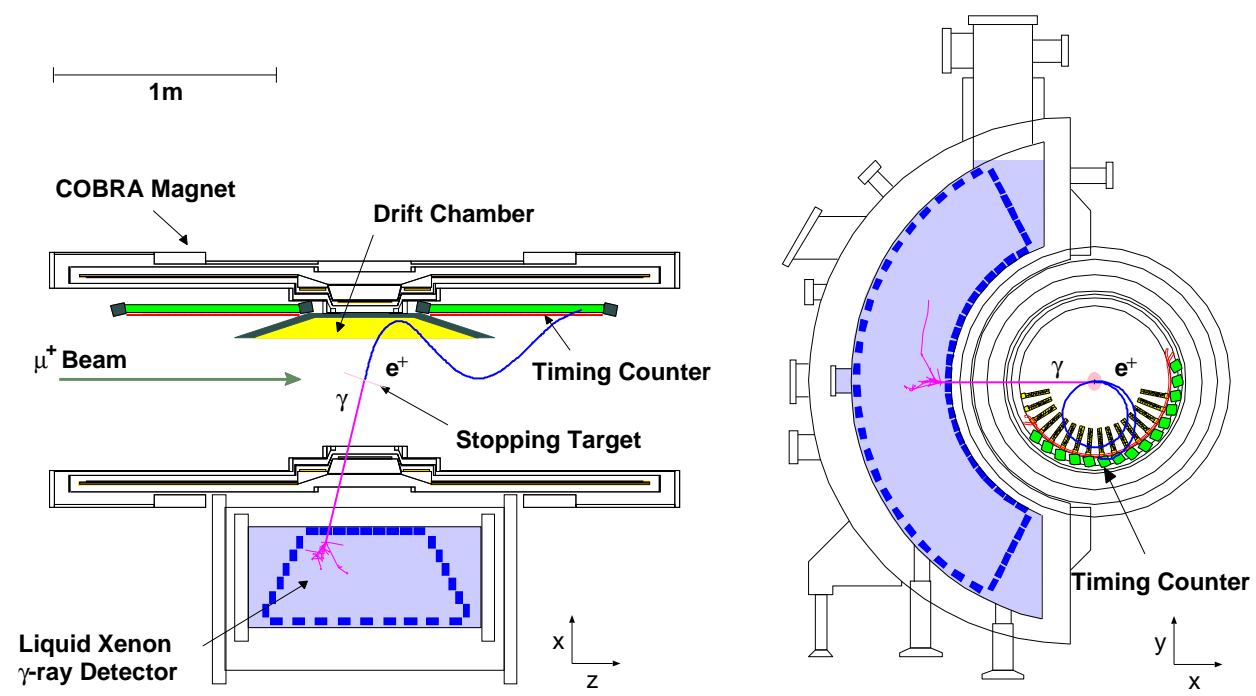

Figure 3: Schematic of MEG detector.

The spectrometer performance was studied using Michel positrons. Resolutions are extracted by using positrons making two turns in DC. The differences of the reconstructed variables by each turn give estimators of resolutions. The energy resolution is well described by a double Gaussian with sigma of 0.39 and $1.71 \mathrm{MeV}$ for core (79\%) and tail (21\%) component, respectively. It is confirmed independently by a fit to the kinematic edge of the Michel spectrum shown in Figure $⿴$. The angular resolutions are evaluated to be $\sigma_{\theta_{e}}=11.2, \sigma_{\phi_{e}}=7.1 \mathrm{mrad}$. The vertex resolution are evaluated to be 2.8 and $2.3 \mathrm{~mm}$ for the beam axis and vertical directions, respectively. These values are cross-checked by looking at the edges in reconstructed vertex distribution corresponding to holes on the target. The performance of the LXe detector is essentially same as that in 2008 . We achieved energy resolution of $2.1 \%(2 \mathrm{~cm}<w), 2.8 \%(1<w<2 \mathrm{~cm}), 3.3 \%(w<1 \mathrm{~cm})$, where $w$ is reconstructed depth from the front face. The position resolutions are evaluated to be 5 and $6 \mathrm{~cm}$ for the direction along the front face and for the depth, respectively. The timing resolution is estimated to be 142 ps by the RD peak in low-energy region shown in Figure $\square$ with extrapolation to the signal energy.

The number of Michel positrons counted simultaneously with the signal with the same analysis cuts is $18096 \times P_{e v \bar{v}}$, where $P_{e v \bar{v}}=1.2 \times 10^{7}$ is a pre-scale factor of Michel trigger. The fraction of Michel spectrum detected in a geometrical acceptance of the spectrometer is calculated to be $f_{e v \bar{v}}^{E}=$ 0.114. Those numbers are used for normalization of the branching fraction of $\mu^{+} \rightarrow \mathrm{e}^{+} \gamma$ decay with small corrections of momentum dependence of efficiencies:

$$
\frac{\mathscr{B}\left(\mu^{+} \rightarrow \mathrm{e}^{+} \gamma\right)}{\mathscr{B}\left(\mu^{+} \rightarrow \mathrm{e}^{+} v \bar{v}\right)}=\frac{N_{s i g}}{N_{e v \bar{v}}} \cdot \frac{f_{e v \bar{v}}^{E}}{P_{e v \bar{v}}} \cdot R_{t r i g} \cdot R_{e} \cdot \varepsilon_{\gamma}=N_{\text {sig }} \times(1.01 \pm 0.08) \times 10^{-12}
$$

where $R_{t r i g}$ and $R_{e}$ are efficiency ratios between signal and Michel positron for trigger and positron reconstruction respectively, and $\varepsilon_{\gamma}=0.58$ is gamma-ray detection efficiency conditional to that the corresponding signal positron is detected. 


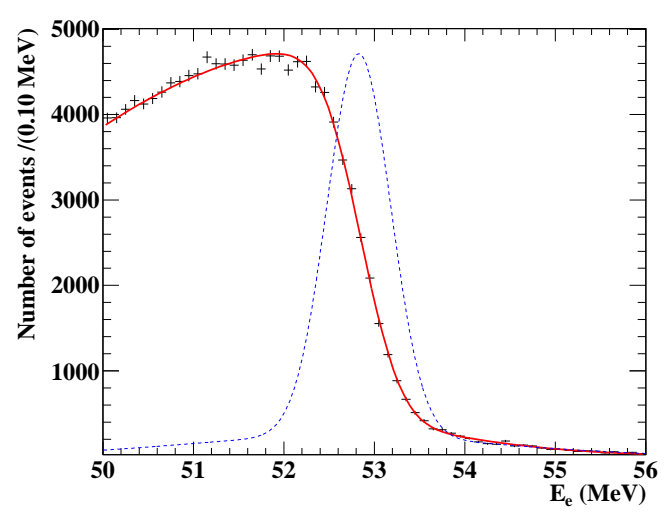

Figure 4: Measured spectrum of positron energy. Kinematic edge was fitted by convolving detector response shown with blue dashed line.

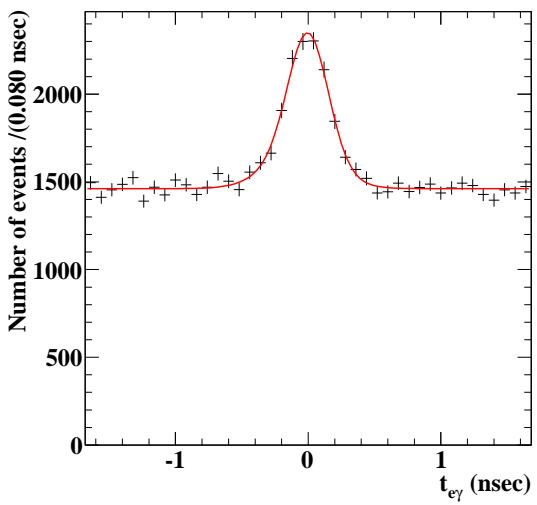

Figure 5: Measured time distribution. The RD peak can be seen on top of a flat floor from accidental background.

\section{Analysis}

Observable variables used to discriminate signal from backgrounds are the following: gamma ray energy $\left(E_{\gamma}\right)$, positron energy $\left(E_{e}\right)$, time difference of the two particles $\left(t_{e \gamma}\right)$ and angle difference between gamma ray direction and reverse direction of positron $\left(\theta_{e \gamma}\right.$ and $\left.\phi_{e \gamma}\right)$. Events around the signal region in $\left(E_{\gamma}, t_{e \gamma}\right)$-plane were hidden until analysis was fixed. The detector calibration and performance evaluation were done using calibration samples and events outside the hidden box (sideband data). The background level in the signal region can be also studied using sideband data: $t_{e \gamma}$-sideband for the accidental one and $E_{\gamma}$-sideband for the RD one.

We estimate the number of signal, RD and accidental background (BG) in a pre-defined analysis window with an unbinned maximum likelihood fit. The analysis window is taken widely enough to estimate the background distribution. An extended maximum likelihood is build as,

$$
\mathscr{L}\left(N_{s i g}, N_{R D}, N_{B G}\right)=\frac{N^{N_{\mathrm{obs}}} e^{-N}}{N_{\mathrm{obs}} !} \prod_{i=1}^{N_{\mathrm{obs}}}\left(\frac{N_{s i g}}{N} \cdot S\left(\vec{x}_{i}\right)+\frac{N_{R D}}{N} \cdot R\left(\vec{x}_{i}\right)+\frac{N_{B G}}{N} \cdot B\left(\vec{x}_{i}\right)\right)
$$

where $\vec{x}_{i}$ is a vector of the five observable variables for the $i$-th event, $N_{s i g}, N_{R D}$ and $N_{B G}$ are number of signal, RD and BG, respectively, while $S, R$ and $B$ are their respective probability density functions (PDFs). $N_{\text {obs }}$ is observed total number of events in the analysis window, while $N=N_{s i g}+N_{R D}+N_{B G}$ is the expected one. The PDFs are obtained from the experimental data described in Sec $⿴$ on event-by-event basis to take into account the position-dependent LXe detector response and tracking quality for each positron. A confidence region is build by the likelihood-ratio ordering principle [ $[\mathrm{W}]$ in the $\left(N_{s i g}, N_{R D}\right)$-plane and the confidence interval on $N_{\text {sig }}$ is extracted from the projection of the region to the $N_{\text {sig }}$-axis.

Here, we define a sensitivity of this search with a mean of upper-limit distribution over an ensemble of toy-MC experiments with null signal. The branching-fraction sensitivity at $90 \% \mathrm{CL}$ is evaluated to be $6.1 \times 10^{-12}$. This is consistent of the results of obtained upper limits of $(4-$ $6) \times 10^{-12}$ when we perform the likelihood analysis on several comparable analysis windows in $t_{e \gamma}$-sideband by shifting the reference of the time difference. 


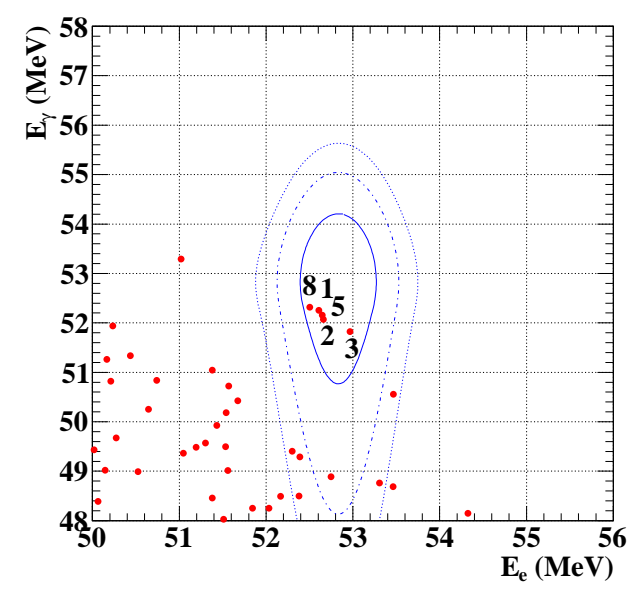

(a)

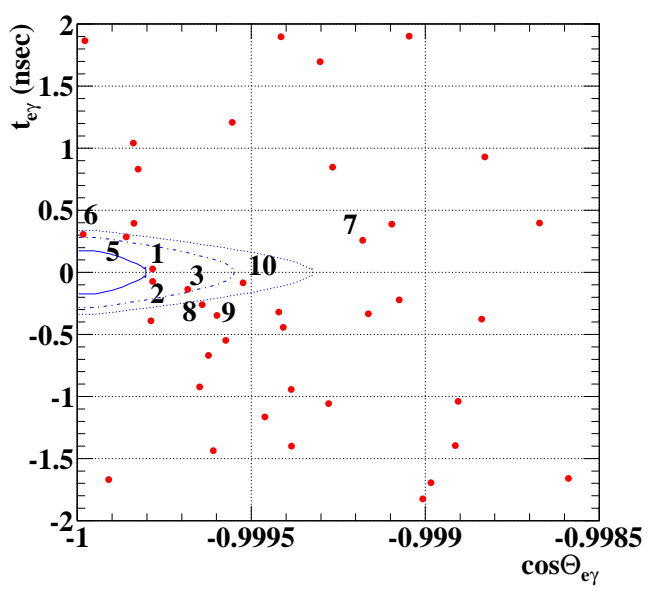

(b)

Figure 6: Event distribution (a) in $E_{e}-E_{\gamma}$ plane after cuts on $\cos \Theta_{e \gamma}$ and $t_{e \gamma}$ with $90 \%$ efficiency for each are applied and (b) in $\cos \Theta_{e \gamma}-t_{e \gamma}$ plane after cuts on $E_{e}$ and $E_{\gamma}$ with $90 \%$ efficiency for each are applied, where $\Theta_{e \gamma}$ is opening angle between the two particle directions. The contours of signal PDFs at 1-, 1.64and $2-\sigma$ are shown. Same events in the two plots are numbered correspondingly by decreasing ranking by the relative signal likelihood $(S /(R+B))$.

We opened the hidden box when all the studies were completed. Figure 6 shows the event distributions inside the box. One to two background events are expected to fall around the signal region according to the background rate evaluated in the sidebands. The events locating close to the signal region were carefully checked and no strange behavior of detectors was found for all those events. The maximum likelihood fit to 370 events in the analysis window, whose result as projections on each variables is shown in Figure $\square$, gives the $N_{\text {sig }}$ best-estimate value at 3.0. That of $N_{R D}=35_{-22}^{+24}$ is consistent with the expected number of $32 \pm 2$ from the $E_{\gamma}$-sideband. The confidence region is constructed by means of toy-MC simulation with taking into account possible systematic effects. The PDFs and normalization factor are fluctuated for each toy-MC experiment in accordance with their uncertainty values. The point of $N_{\text {sig }}=0$ is included in the $90 \%$-confidence interval, and an upper limit is calculated to be $N_{\text {sig }}<14.5$. This, together with the normalization Eq. (لل]), yields an upper limit on the branching fraction,

$$
\frac{\mathscr{B}\left(\mu^{+} \rightarrow \mathrm{e}^{+} \gamma\right)}{\mathscr{B}\left(\mu^{+} \rightarrow \mathrm{e}^{+} v \bar{v}\right)}<1.5 \times 10^{-11} \quad(90 \% \mathrm{CL})
$$

Three independent analyses with different statistical approaches were performed to check the analysis, and gave consistent results.

\section{Conclustion}

A search for the lepton flavor violating decay $\mu^{+} \rightarrow \mathrm{e}^{+} \gamma$ based on data of MEG second year run was described. The sensitivity is evaluated to be $6.1 \times 10^{-12}$, which is twice better than the current limit. The fix of the problem on DC system appeared in 2008 run enabled us to achieve this 

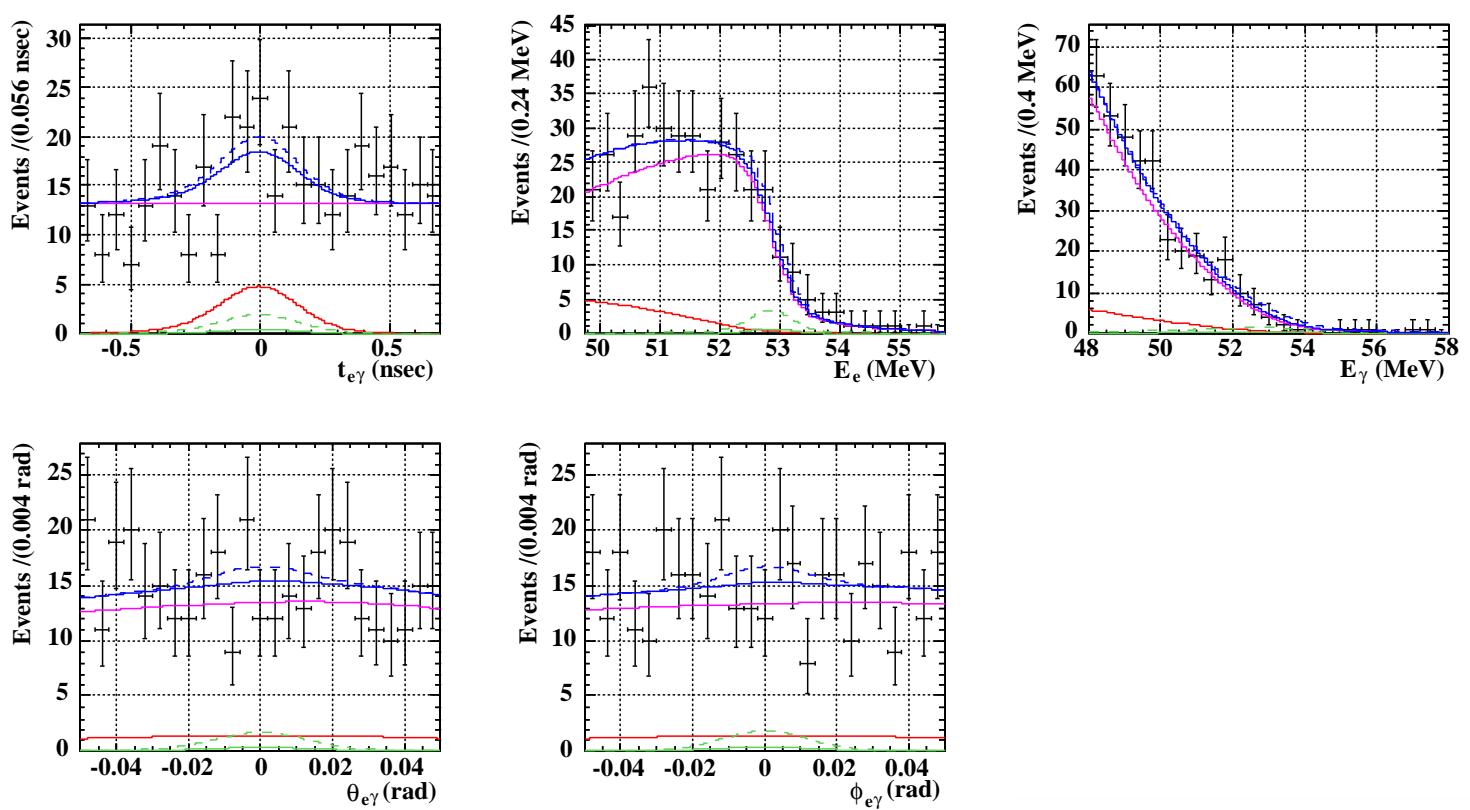

Figure 7: Event distributions in the fit region projected on each variable and result of the maximum likelihood fit. The green, red and magenta curves show signal, RD and BG PDFs, respectively, and blue for the total. The solid lines show the best-fit distributions, while the dashed ones show those with $N_{\text {sig }}$ at the $90 \%$ upper limit.

unexplored-region sensitivity even with a shorter period of data acquisition. A preliminary analysis sets an upper limit on the branching fraction, $\mathscr{B}\left(\mu^{+} \rightarrow \mathrm{e}^{+} \gamma\right) / \mathscr{B}\left(\mu^{+} \rightarrow \mathrm{e}^{+} v \bar{v}\right)<1.5 \times 10^{-11}$ at 90\% CL. MEG resumed data taking in August 2010 and has been accumulating statistics with stable detector condition. It will run at least until the end of 2012 to reach our goal of $(2-3) \times 10^{-13}$ sensitivity.

\section{References}

[1] R. Barbieri, L. Hall and A. Strumia, Nucl. Phys. B 455 (1995) 219.

[2] J. Hisano et al., Phys. Lett. B 391 (1997) 341.

[3] M. L. Brooks et al. [MEGA collaboration], Phys. Rev. Lett. 83 (1999) 1521.

[4] T. Mori et al., Rerearch proposal to PSI, R-99-05 (1999), http://meg.web.psi.ch/docs/.

[5] J. Adam et al. [MEG collaboration], Nucl. Phys. B 834 (2010) 1.

[6] Y. Kuno, A. Maki and Y. Okada, Phys. Rev. D 55 (1997) R2517.

[7] G. J. Feldman and R. D. Cousins, Phys. Rev. D 57 (1998) 3873. 